\title{
TARGETED THERAPIES \\ Bevacizumab and chemotherapy do not increase the risk of VTEs
}

Venous thromboembolisms (VTEs) are associated with a high level of morbidity and mortality in patients with cancer. The risk of developing a VTE can also be affected by the type of cancer, tumor burden, chemotherapy regimen and other factors such as patient comorbidities. The role of the VEGF inhibitor bevacizumab in contributing to this problem is controversial. Although one metaanalysis showed an increased risk of VTEs associated with bevacizumab, this finding has not been confirmed in other studies.

To quantify the risk of VTE associated with bevacizumab, Herbert Hurwitz and coauthors performed a meta-analysis. As Hurwitz explains, "our study evaluated individual patient data from over 6,000 patients from across 10 phase III clinical trials. This is the largest and most rigorous analysis to date on this topic." Three clinical questions were addressed in the study: first, does bevacizumab increase the risk of VTEs; second, what are the risk factors for developing a VTE and do these differ in bevacizumab compared with control-treated patients; third, is the risk of serious bleeding in those who develop a VTE increased with bevacizumab use. The meta-analysis showed there was no significant increase in the risk of VTE related to bevacizumab. This finding was consistent across all tumor types assessed and did not differ according to the chemotherapy regimen used. Common risk factors for VTEs and serious bleeding during anticoagulation therapy were not affected by bevacizumab. Thus, the risk for developing VTEs is mainly driven by tumor and host factors. Hurwitz notes, "bevacizumab, when added to chemotherapy, did not increase the risk of VTEs. Our results confirmed the importance of several risk modifiers, including tumor type and several patientspecific factors, such as age, performance status, and prior thromboembolic events". In terms of future studies, Hurwitz

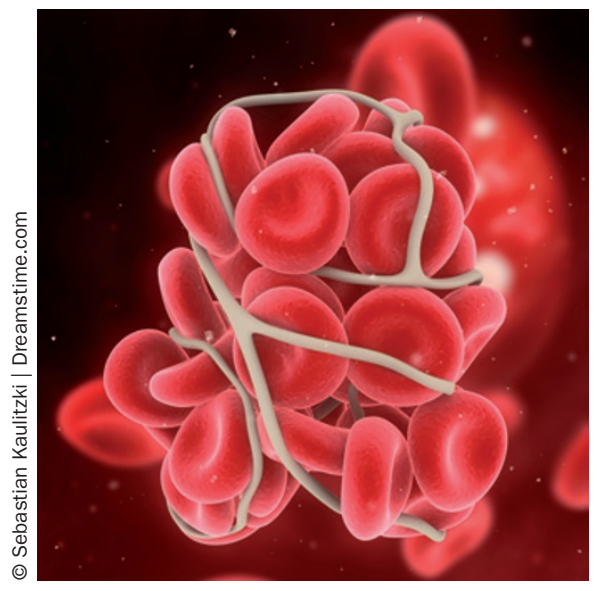

comments, "hopefully similar analyses using patient-specific data from many phase III trials will be performed for other known complications of bevacizumab, such as arterial thromboembolic events and gastrointestinal perforations."

Lisa Hutchinson

Original article Hurwitz, H. I. et al. Venous thromboembolic events with chemotherapy plus bevacizumab: a pooled analysis of patients in randomized phase II and III studies. J. Clin. Oncol. doi:10.1200/JC0.2010.32.3220 